09

\title{
Фототермическая ИК-радиометрия \\ в экспериментальных исследованиях пироэлектрических свойств объемных материалов
}

\author{
(C) С.Е. Александров, Г.А. Гаврилов, А.А. Капралов, \\ К.Л. Муратиков Ф, Г.Ю. Сотникова
}

Физико-технический институт им. А.Ф. Иофффе РАН, Санкт-Петербург

『E-mail: klm.holo@mail.ioffe.ru

Поступило в Редакцию 12 июля 2017 г.

Предложен простой и эффективный метод определения величины пирокоэффициента сегнетоэлектрических и пироэлектрических объемных материалов с учетом теплообмена образца с окружающей средой, основанный на радиометрической регистрации динамики изменения температуры его поверхности при воздействии на нее лазерного излучения в виде временно́й ступеньки.

DOI: 10.21883/PJTF.2017.23.45279.16966

Изучению пироэлектрических свойств сегнетоэлектрических и пироэлектрических материалов уделяется серьезное внимание [1]. Пироэлектрический коэффициент является важнейшей характеристикой материала для создания радиационных сенсоров и накопителей энергии (energy harvesters) [2,3]. В настоящее время при проведении пироэлектрических экспериментов широко используются нестационарные методы нагрева образцов с применением лазерного излучения [4-7]. Применяются как методы импульсного теплового воздействия (thermal pulse (TP) method) [7], так и методы с периодическим (laser intensity modulation method (LIMM)) тепловым воздействием [4-6].

Большинство пироэлектрических экспериментов проводится в условиях, когда лицевые поверхности образцов находятся в контакте с окружающей газовой средой (обычно воздухом). В этом случае важным теплофизическим параметром является коэффициент теплоотдачи $H$, характеризующий эффективность теплообмена образца с окружающей средой. Коэффициент теплоотдачи зависит от качества обработки поверхности образца и от теплообмена образца с окружающей средой [8]. 
Значение коэффициента $H$ трудно оценить экспериментально. При проведении TP- и LIMM-пироэлектрических экспериментов с сегнетоэлектрическими материалами теплообмен образца обычно считают пренебрежимо малым и в расчетах полагают $H=0$ [5]. Вместе с тем даже в условиях вакуума коэффициент теплоотдачи в результате теплового излучения имеет отличное от нуля значение [8]. Отсутствие знаний об истинном значении коэффициента $H$ является одним из источников погрешности в определении пирокоэффициента одного и того же материала в различных экспериментальных установках [1]. Таким образом, при проведении реальных измерений важно иметь методики, позволяющие независимым образом измерять коэффициент теплообмена и пироэлектрические характеристики образца в условиях одного и того же эксперимента. Такой подход позволяет определять пирокоэффициент с учетом реального теплообмена образца с окружающей средой и дает возможность оценить степень влияния допущения $H=0$ на его значения. В связи с этим основной целью настоящей работы является отработка методики измерения пирокоэффициента объемных материалов с учетом теплообмена образца с окружающей средой, а также оценка степени влияния на его величину допущения об отсутствии теплообмена.

Для решения поставленной задачи использовалась методика измерения коэффициента $H$ для исследуемого образца [9]. Для исключения влияния контактных процессов на температуру образца в работе применялись пирометрические методы измерения температуры. Регистрация теплового излучения от образцов осуществлялась с помощью быстродействующих спектрально-селективных иммерсионных фотодиодов (ФД) среднего ИК-диапазона спектра $(2-5 \mu \mathrm{m})$, работающих без принудительного охлаждения [10].

В [9] было показано, что при длительностях засветки $\tau$, удовлетворяющих условию $\sqrt{(\kappa \tau)} \gg L(\kappa-$ коэффициент температуропроводности материала, $L$ - толщина образца), температуры лицевой и тыльной поверхностей образца имеют близкие значения и их можно найти с помощью соотношения

$$
T_{s}(t)=\frac{(1-R) I_{0}}{\rho C L} \int_{t_{1}}^{t} d t^{\prime} e^{-\frac{2 H}{\rho C L}\left(t-t^{\prime}\right)} f\left(t^{\prime}\right)+T_{0},
$$

где $t_{1}$ - время начала лазерного воздействия, $\rho$ - плотность материала образца, $C$ - его удельная теплоемкость, $I_{0}-$ интенсивность излучения лазера, $R$ - коэффициент отражения света поверхностью образца,

Письма в ЖТФ, 2017, том 43, вып. 23 
$H-$ коэффициент теплоотдачи, $T_{0}-$ температура окружающей среды, $f(t)$ - функция, описывающая временну́ю характеристику лазерного воздействия.

Выражение (1) при известных параметрах образца, лазерного излучения и измеренной зависимости $T_{s}(t)$ может быть использовано для определения коэффициента теплоотдачи $H$. В соответствии с (1) учет теплообмена образца с окружающей средой приводит к появлению времени релаксации $\tau_{t h}=\frac{\rho C L}{2 H}$. Важно, что величина коэффициента $H$ является характеристикой конкретного эксперимента, она зависит от качества обработки поверхности образца и условий его теплообмена с окружающей средой [8].

При воздействии на образец лазерным излучением, для которого функция $f(t)$ представляет собой единичный скачок интенсивности лазерного излучения в момент времени $t>t_{1}$, поведение температуры образца при указанных выше условиях в соответствии с (1) определяется равенством

$$
T_{S}(t)=\frac{I_{0}}{2 H}\left(1-e^{-\frac{2 H}{\rho C L}\left(t-t_{1}\right)}\right)+T_{0} .
$$

Знание зависимости температуры образца от времени позволяет рассчитать ожидаемый пироток образца. При условии однородности пироэффекта по толщине образца в нашем приближении он может быть записан в виде

$$
i_{p}(t)=p A \frac{d T_{s}}{d t}=p A \frac{I_{0}}{\rho C L} e^{-\frac{2 H}{\rho C L} t},
$$

где $A-$ площадь электродов образца, $p$ - интегральный (по толщине образца) пирокоэффициент материала.

В соответствии с равенствами (2) и (3) деление выражения (3) на производную по времени от температуры (2) дает значение коэффициента $p A$ для времен, удовлетворяющих условию $\sqrt{(\kappa \tau)} \gg L$. Таким образом, выражения (2) и (3) показывают, что независимые измерения температуры и пиротока при известных теплофизических параметрах образца позволяют определять пирокоэффициент исследуемого материала с учетом реально присутствующего конвективного теплообмена с окружающей средой. Полученные выражения также позволяют оценить погрешность измерения пирокоэффициента образца при пренебрежении его теплообменом с окружающей средой.

Письма в ЖТФ, 2017, том 43, вып. 23 
В рамках настоящей работы экспериментальные исследования проводились на объемном образце хорошо изученного сегнетоэлектрического монокристалла танталата лития $\left(\mathrm{LiTaO}_{3}\right)$. Исследовался тестовый образец $\mathrm{LiTaO}_{3} \mathrm{Z}$-среза, который представлял собой диск диаметром $10 \mathrm{~mm}$ и толщиной $L=0.615 \mathrm{~mm}$. Электроды полностью занимали поверхности диска. Для увеличения коэффициента поглощения лазерного излучения и повышения излучательной способности они покрывались тонким слоем поглощающего материала. Источником возбуждения тепловых волн в образце являлся полупроводниковый лазер с длиной волны $\lambda=638 \mathrm{~nm}$ и регулируемой мощностью излучения $0.05-0.3 \mathrm{~W}$, допускающий модуляцию мощности излучения от внешнего генератора сигналов произвольной формы. Лазерный пучок различной мощности равномерно засвечивал лицевую поверхность образца, свободно лежащего на кольцевом держателе внутри кюветы с сапфировыми окнами, прозрачными для возбуждающего лазерного излучения и регистрируемого теплового излучения. Отвод тепла от торцов образца не играл существенной роли, так как их суммарная площадь значительно меньше площади лицевых поверхностей.

Для контроля температуры поверхностей использовались ФД типа PD42SrNB, выпускаемые OOO „ИоффеЛЕД“, чувствительные в области $\Delta \lambda=3.4-4.4 \mu \mathrm{m}[11]$. ФД использовались без дополнительных оптических элементов. Сбор теплового излучения осуществлялся в

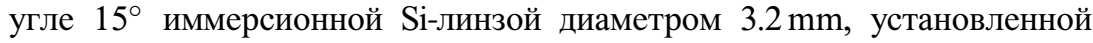
непосредственно на чип ФД. Электронное обрамление ФД обеспечивало детектирование сигнала в полосе $3 \mathrm{kHz}$ для температур выше $20^{\circ} \mathrm{C}$ с точностью не хуже $0.1^{\circ} \mathrm{C}$. Установка содержала также кремниевый ФД для регистрации формы возбуждающего лазерного излучения и схему детектирования и усиления пиротока с коэффициентом преобразования $2.64 \cdot 10^{7} \mathrm{~A} / \mathrm{V}$. Все регистрируемые сигналы оцифровывались (12 бит) и передавались на ПК, где обеспечивались on-line расчет, вывод на экран монитора и запись в файл информации о динамике регистрируемых сигналов с временны́м разрешением $1.5 \mathrm{~ms}$.

На рис. 1 представлены экспериментальные данные для изменения температуры тыльной поверхности образца при воздействии на его лицевую поверхность лазерного излучения в виде единичного скачка различной амплитуды: $P \sim 15$ и $\sim 60 \mathrm{~mW}$. Переходные характеристики для температуры поверхности образца хорошо аппроксимируются экспоненциальной функцией вида (2) (сплошные линии) и позволяют

Письма в ЖТФ, 2017, том 43, вып. 23 


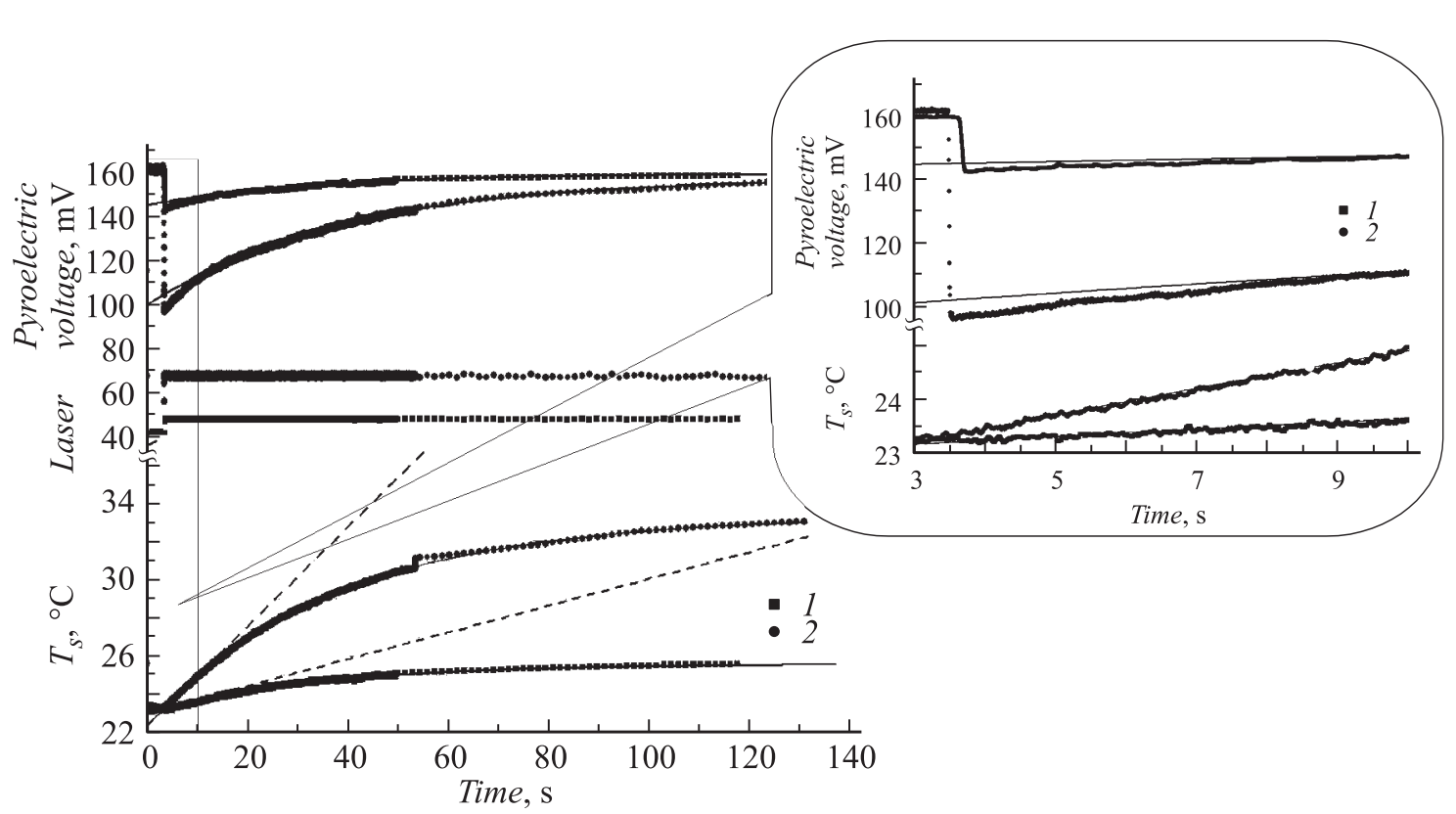

Рис. 1. Экспериментальные точки и расчетные кривые (сплошные линии) для временны́х зависимостей температуры поверхности (внизу) и пироэлектрического отклика (вверху) образца $\mathrm{LiTaO}_{3}$ при воздействии на него лазерного излучения в виде временно́й ступеньки (в середине) различной амплитуды: $P \approx 15(1)$ и $\sim 60 \mathrm{MW}(2)$. Штриховыми линиями показаны ожидаемые зависимости для температуры поверхности образца в предположении, что тепловыми потерями можно пренебречь $(H=0)$. 
рассчитать коэффициент теплоотдачи $H$. В результате расчетов было получено значение $H=28.6 \mathrm{~W} /\left(\mathrm{m}^{2} \cdot \mathrm{K}\right)$. Соответствующая данному значению постоянная времени релаксации составляла $\tau_{t h} \approx 50 \mathrm{~s}$. При толщине образца $0.615 \mathrm{~mm}$ условие $\sqrt{(\kappa \tau)} \gg L$ обеспечивает выполнение выражения (2) для времен $\tau>1-2 \mathrm{~s}$. Для $\mathrm{LiTaO}_{3}$ значения теплофизических коэффициентов теплопроводности и температуропроводности считались известными и равными соответственно $K=3 \mathrm{~W} /(\mathrm{m} \cdot \mathrm{K})$ и $\kappa \approx 0.94 \cdot 10^{-6} \mathrm{~m}^{2} / \mathrm{s}$ [12]. Однако следует отметить, что при необходимости лазерные термоволновые методики позволяют определить и теплофизические параметры образца [13-15].

Начальные участки переходных характеристик на рис. 1 представлены с временны́ разрешением $1.5 \mathrm{~ms}$, а на участках $\mathrm{c} t>\tau_{t h}-\mathrm{c}$ временни́м разрешением $1.5 \mathrm{~s}$. Наблюдается хорошее согласие (коэффициент корреляции 0.98-0.99) экспериментальных данных для температуры поверхности образца с экспоненциальной зависимостью (2) во всем временно́м интервале (более $2 \mathrm{~min}$ ). Однако для пиротока на начальном участке при $t<\tau_{0} \approx 5-6 \mathrm{~s}$ имеет место заметное расхождение экспериментальных данных и расчетных значений, полученных в соответствии с (3). Это может быть связано с наличием других переходных процессов в образце, в частности с третичным пироэффектом [1].

На рис. 2 представлены временнб́е зависимости величины пирокоэффициента, рассчитанные с использованием синхронно регистрируемых экспериментальных значений пиротока и температуры поверхности образца. Данные представлены для двух экспериментов с различной мощностью лазерной засветки: $P \sim 60$ и $\sim 90 \mathrm{~mW}$, для которых обеспечивается высокое значение отношения сигнал/шум регистрируемых сигналов в течение длительного времени измерений (не менее $30 \mathrm{~s}$ ). Наблюдается хорошее согласие расчетных данных, полученных на основе экспериментов с различным уровнем засветки. На этом же рисунке представлены расчетные временны́е зависимости величины пирокоэффициента, полученные с использованием текущих значений пироэлектрического сигнала в предположении, что тепловыми потерями можно пренебречь $(H=0)$. При этом температура поверхности должна изменяться линейно с постоянной скоростью, зависящей только от уровня лазерной засветки.

Часто используемое на практике измерение пирокоэффициента непосредственно после подачи лазерного излучения на образец дает значение $p=1.2 \cdot 10^{-4} \mathrm{C} /\left(\mathrm{m}^{2} \cdot \mathrm{K}\right)$. Однако, как видно из вставки к 


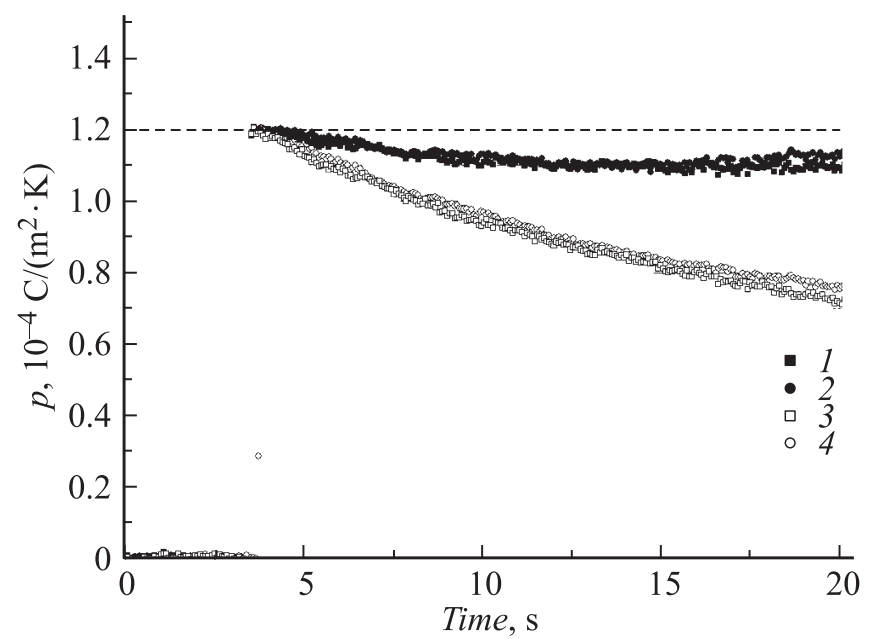

Рис. 2. Значения пирокоэффициента, рассчитанные с использованием синхронно регистрируемых экспериментальных данных для пироэлектрического отклика и температуры поверхности образца при двух типах лазерного воздействия в виде временно́й ступеньки различной мощности: $P \sim 60(1)$ и $\sim 90 \mathrm{~mW}(2)$. Нижние кривые соответствуют расчетным зависимостям величины пирокоэффициента, полученным с использованием текущих значений пироэлектрического сигнала при тех же уровнях лазерного воздействия $(P \sim 60(3)$ и $\sim 90 \mathrm{~mW}(4))$ в предположении, что тепловыми потерями можно пренебречь $(H=0)$.

рис. 1 , на начальном участке, при $t<\tau_{0}$, наблюдается отклонение пиротока от ожидаемой теоретической зависимости, связанное с наличием различных переходных процессов в образце, что приводит к завышенному значению пирокоэффициента (например, вследствие третичного пироэффекта). Для времен $t>\tau_{0}$ режим измерений пирокоэффициента можно считать квазистационарным. В соответствии с (3) для расчета пирокоэффициента из измерений пиротока $i_{p}(t)$ необходимо домножать его значение на экспоненту, в показатель которой входит $H$. При обработке экспериментальных данных для $\tau \approx \tau_{t h}$ нами использовалось значение $H$, полученное для данного образца из радиометрических измерений температуры. При временах $t \ll \tau_{t h}$ приближение $H=0$ не вносит существенной ошибки в такую процедуру, но ограничивает

6* Письма в ЖТФ, 2017, том 43, вып. 23 
предельное время усреднения пирокоэффициента. При этом наличие различных переходных процессов в данной области не позволяет считать их полностью достоверными.

Измерения в квазистационарных условиях при $t \approx \tau_{t h}$ не только являются более надежными, но и обеспечивают бо́льшую точность, так как максимальное время усреднения для пирокоэффициента ограничивается только измеримостью пиротока с достаточно высоким отношением сигнал/шум. Так, в соответствии с рис. 2 обработка экспериментальных результатов с помощью выражений (2) и (3) за период 10-20 s дает практически постоянное значение пирокоэффициента $p=(1.11 \pm 0.01) \cdot 10^{-4}\left(\mathrm{C} / \mathrm{m}^{2} \cdot \mathrm{K}\right)$.

Таким образом, полученные результаты показывают, что лазерные теплофизические методики с засветкой образца излучением в виде временно́й ступеньки при прямом бесконтактном измерении температуры его поверхности могут быть использованы для определения пирокоэффициента объемных материалов и не требуют использования специального калориметрического оборудования. При этом учитывается наличие переходных процессов непосредственно после начала засветки и производится оценка пирокоэффициентов с учетом теплоотвода в окружающую среду.

Авторы выражают благодарность сотрудникам ООО „ИоффеЛЕД“, предоставившим ФД среднего ИК-диапазона для создания экспериментальной измерительной установки.

\section{Список литературы}

[1] Jachalke S., Mehner E., Stocker H. et al. //Appl. Phys. Rev. 2017. V. 4. Iss. 2. P. 021303.

[2] Sebald G., Lefeuvre E., Guyomar D. // IEEE Trans. Ultrason. Ferroelectrics Frequency Control. 2008. V. 55. Iss. 3. P. 538-551.

[3] Александров С.Е., Гаврилов Г.А., Капралов А.А. и др. // ЖТФ. 2004. Т. 74. B. 9. C. $72-76$.

[4] Lang S.B., Das-Cupta D.K. // J. Appl. Phys. 1986. V. 59. Iss. 6. P. 2151-2160.

[5] Bauer S., Ploss B. // J. Appl. Phys. 1990. V. 68. Iss. 12. P. 6361-6367.

[6] Biryukov S.V., Sotnikov A.V., Weihnacht M. // Integrated Ferroelectrics. 2001. V. 35. P. 67-75.

[7] Bloss P., DeReggi A.S., Schafer H. // Phys. Rev. B. 2000. V. 62. Iss. 12. P. $8517-$ 8530. 
[8] Кухлинг Х. Справочник по физике. М.: Мир, 1982. 520 с.

[9] Александров С.Е., Гаврилов Г.А., Капралов А.А. и др. // Письма в ЖТФ. 2017. T. 43. B. 14. C. 104-110.

[10] Sotnikova G.Yu., Aleksandrov S.E., Gavrilov G.A. // Optical sensors. 2011 Proc. SPIE. 2011. V. 8073. P. 80731A.

[11] www.ioffeled.com

[12] Акустические кристаллы. Справочник / Под ред. М.П. Шаскольской. М.: Наука, 1982. $632 \mathrm{c}$.

[13] Glazov A.L., Muratikov K.L. // Opt. Eng. 1997. V. 36. N 2. P. 358-362.

[14] Глазов А.Л., Козлов В.А, Муратиков К.Л. // Письма в ЖТФ. 2011. Т. 37. B. 24. C. $16-25$.

[15] Глазов А.Л., Калиновский В.С., Контрош Е.В., Муратиков К.Л. // Письма в ЖТФ. 2016. Т. 42. В. 11. С. 33-40. 